\title{
A SYNDROME OF CONTINUOUS MUSCLE-FIBRE ACTIVITY
}

\author{
BY
}

\author{
HYAM ISAACS*
}

\section{Johannesburg}

The purpose of this paper is to describe two cases of a hitherto unknown syndrome superficially resembling myotonia. Detailed studies have helped to separate this syndrome of continuous musclefibre activity from a group of 100 myotonic subjects, and it will be referred to in this paper as the 'syndrome of continuous muscle-fibre activity'. This descriptive label has been chosen, though the more imaginative may favour 'armadillo disease', a name by which these cases are likened to the slow moving, armour-plated quadruped.

\section{Case Reports}

Case 1.-M.P., aged 12, was referred for investigation with a complaint of stiffness of the muscles which had been progressive over the past three years. The patient (Fig. 1), according to his parents, was reasonably normal up to the age of 9 years, quite able to run about and compete with his friends. They stated, however, that from the age of 5 years he had some difficulty in walking

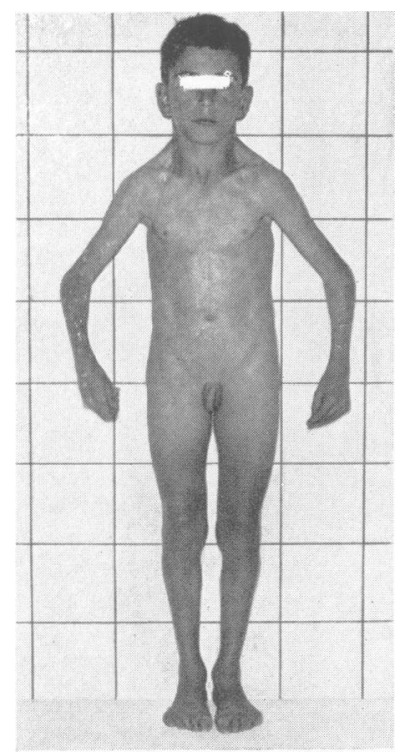

Fig. 1.-Note stiff posture and contraction of muscles (Case 1); contraction of trapezius is not be be mistaken for webbing. and for the past three years he had been severely incapacitated by increasing muscle stiffness, present night and day. The stiffness was increased by voluntary muscle contraction, particularly obvious after the third and fourth movements after which the condition improved and he was able to use his limbs more freely for a time, before they stiffened up again despite continued movement. At times he has had the greatest difficulty with the initial movements and occasionally complained of stiffness of the jaw and tongue while eating and talking. His speech had not been affected but he had at times experienced difficulty in breathing due to stiffness of the chest. Defaecation and micturition were normal. He sweated a great deal and always felt warm. There was no family history of muscle disease; his parents were alive and well and showed no evidence of myopathy. His past illnesses included measles and chickenpox, from which he recovered in the usual time without complications and which were not related to the onset of the present difficulties.

On examination, the patient was found to be underweight and undersized for his 12 years, his height being 48 in., span 47 in., and weight $45 \mathrm{lb}$. Pulse rate was $124 /$ minute, blood pressure $120 / 80 \mathrm{~mm}$. Hg. The heart was normal. The abdominal wall was extremely tense but no hepatosplenomegaly or masses were felt. The genitalia were small, the testes pea-sized and soft. The child was intellectually and emotionally normal. Tendon reflexes were impossible to elicit, but the plantar response was normal. Motor power was generally poor. The cranial nerves were intact. Sensation and coordination seemed normal. His posture was stiff and all muscles were in a state of persistent contraction. The chest moved poorly with respiration; there was hardly any subcutaneous tissue; the muscles were well-defined and firm.

All muscles showed evidence of fasciculation and were weaker than normal. The weakness affected particularly the extensor muscles of the forearms, arms, extensor and peroneal muscles of the legs, and the small muscles of the hands and feet. He walked with extreme difficulty in a rigid manner. The degree of stiffness was more marked in the distal than proximal muscles, being unaffected by the warmth of summer or the cold of winter. The patient was constantly covered by a thin film of perspiration and had a persistent tachycardia of 120 /minute with the body tempcrature averaging $99^{\circ} \mathrm{F}$. The upper limbs could only be fully extended passively and then with difficulty as it appeared that some degree of contracture had occurred. Passive extension was quite painless.

Radiographs of the skull, chest, and spine revealed no 
TABLE I

RESULTS OF LABORATORY INVESTIGATIONS

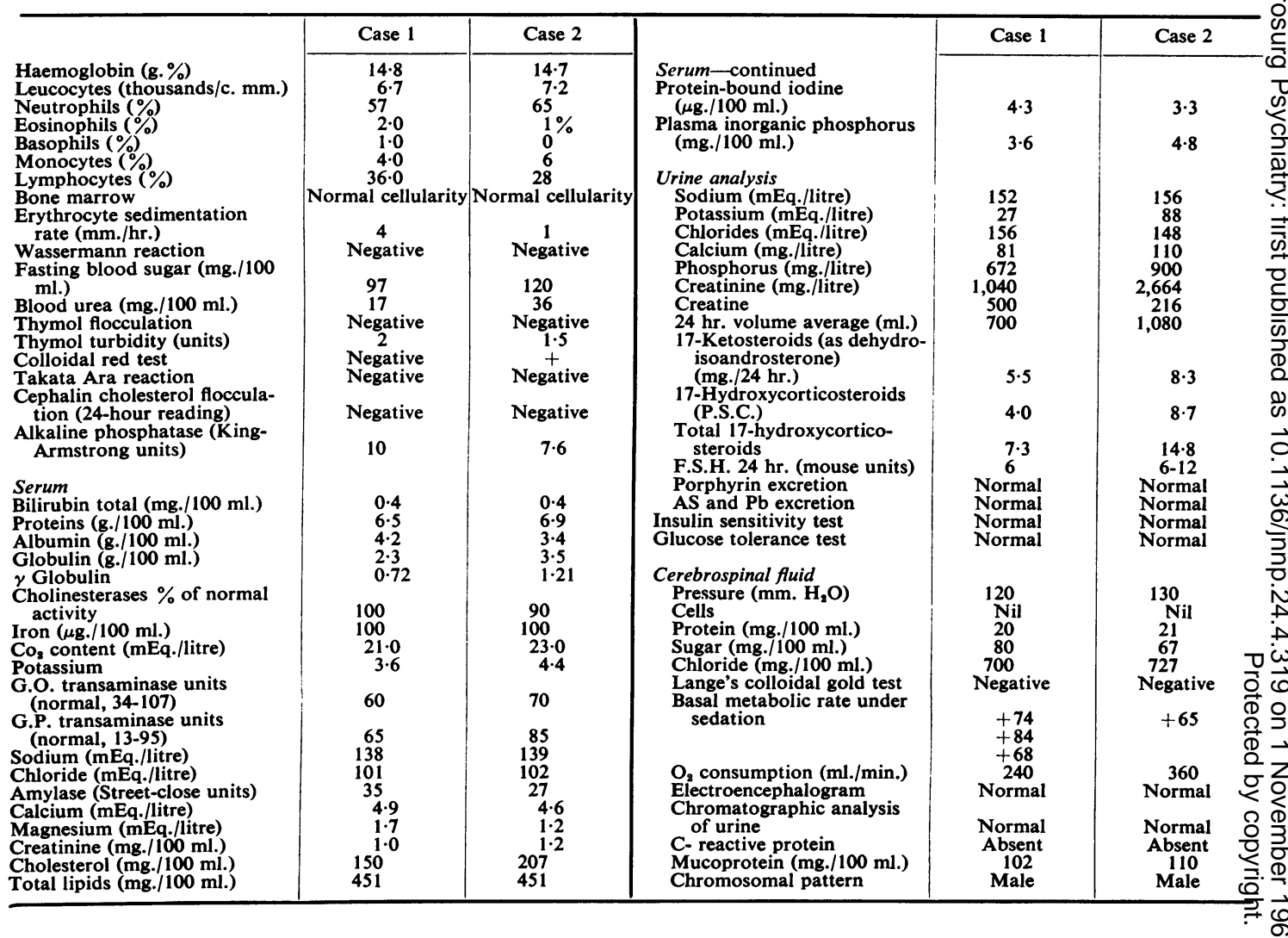

abnormality. Electrocardiograms appeared normal but were difficult to interpret because of the interference from the contracting muscles. The metabolic studies are listed in Table $\mathrm{I}$.

A kymographic recording of the rate of contraction of the muscles of the hand and forearm is shown in Fig. 2. Electromyography carried out with concentric needle electrodes recorded a state of constant rapid dysrhythmic discharge of independent muscle fibres (Fig. 3). The spontaneous activity was increased by voluntary contraction, an increase which peristed for \pm 30 seconds after this stimulus had ceased. This period was followed by a curious period of reduced activity lasting for \pm 10 seconds. This relatively silent period could be interrupted at any stage by further voluntary effort. The electrical response to percussion was normal and did not aggravate the discharge. Electrical stimulation of the nerve, as with voluntary contraction, aggravated the condition. The spontaneous discharge persisted despite local nerve block, but disappeared locally after infiltrating the muscles with procaine. General anaesthesia with pentothal had no effect, but blocking and depolarization of the motor endplate with curare and succinylcholine abolished the spontaneous discharge and the muscle relaxed.

Several muscle biopsy specimens were obtained from proxymal and distal muscles (Fig. 4) which revealed a slight degree of variation in fibre size together with some proliferation of sarcolemmal nuclei, which tended to occur in chains, especially in relation to some of the small fibres. Most of the nuclei were dark and in some places formed small knots. There was a doubtful increase in endomysal collagen and fat.

No improvement occurred following upon the administration of quinidine, procaine amide, cortisone, or potassium depletion by exchange resins. Each of these was given for periods of at least a month in doses pushed to the point of maximum tolerance. A short-lived improvement followed injections of Dimercaprol.

Progress.-Over the next two years the patient was further investigated during the school holidays whenever possible. His condition appeared static; splints were made and applied at night to prevent flexion deformities at the elbows and wrists and to counteract the developing pes cavus. Growth remained retarded, muscle bulk had diminished, and he had several brief attacks of acute respiratory embarrassment due to chest and diaphragmatic stiffness.

After the successful treatment of Case 2 this patient was again admitted to hospital and treated in a similar fashion with dramatic results. 
Fig. 2.-Kymographic tracings with the drum rotating from left to right, demonstrating the slow voluntary contraction and relaxation of the forearm muscles in the act of making a fist.

Fig. 3.-In normal muscle there is no electrical activity at rest. Note continuous dysrhythmic discharge of single muscle fibres at rest.

Fig. 4.-Muscle biopsy from left deltoid showing variations in fibre size, clumping of dark staining nuclei, row of sercolemmal nuclei, and atrophic fibres.

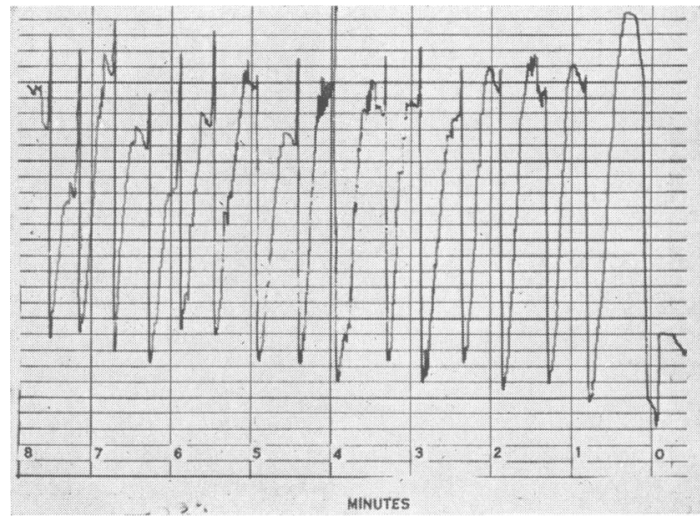

FIG. 2

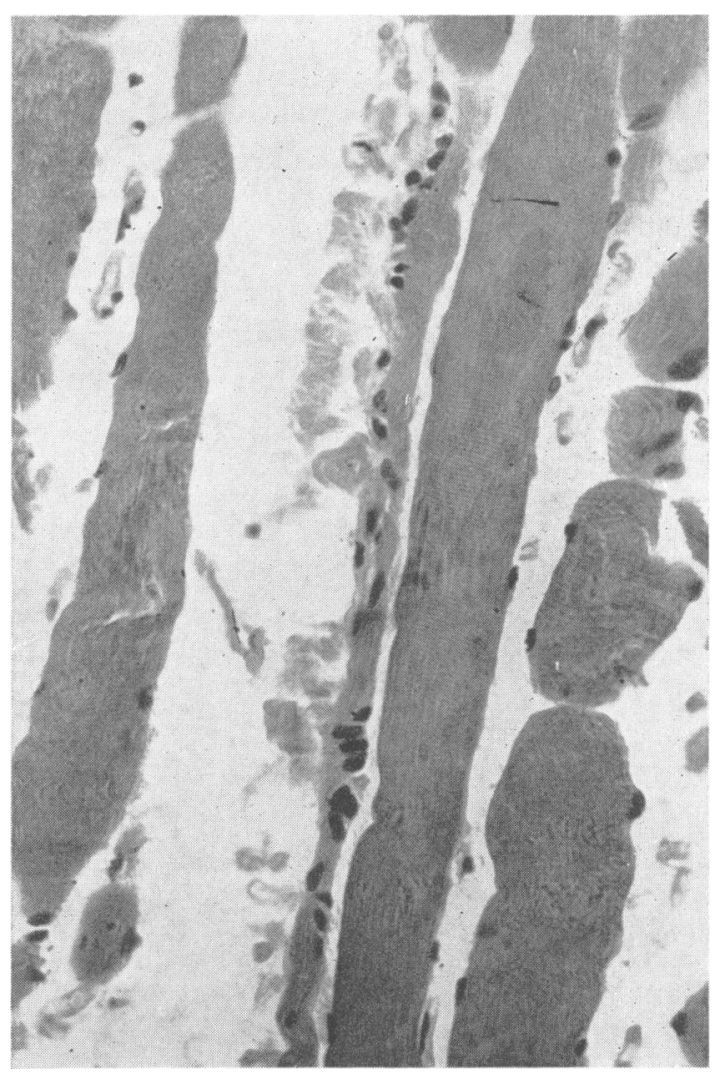

FIG. 4

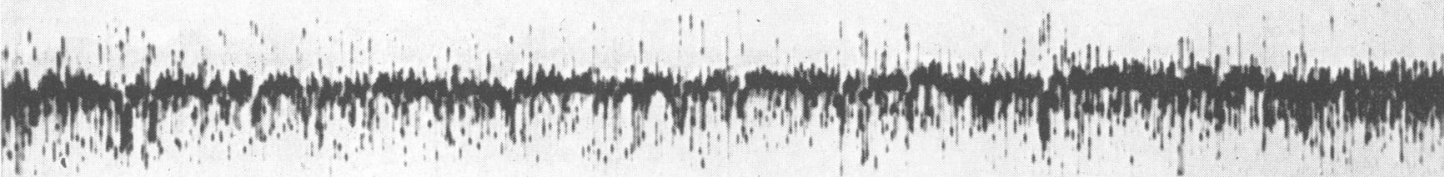

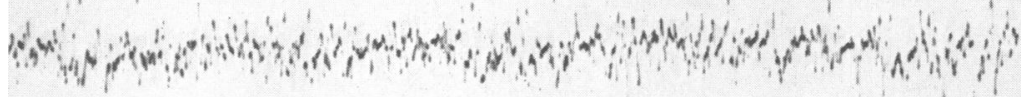

AT REST

FIG. 3 


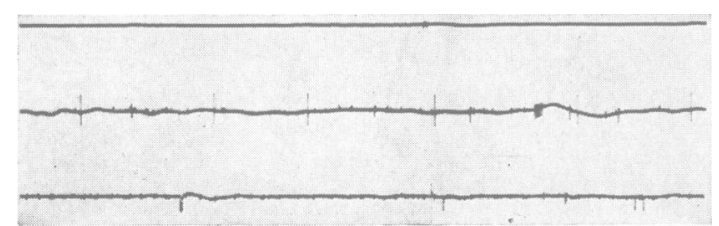

Fig. 5.-A three-channel simultaneous electromyographic recording of first dorsal interosseous (top), extensor communis (middle) and flexor profundus (bottom) muscles at rest, showing marked reduction in activity after three days' therapy in Case 1.

The patient's movement is now quite free and his muscle strength improves daily, aided by intensive physiotherapy. The temperature remains normal, the B.M.R. has fallen to the normal level of +5 and the oxygen consumption is now $160 \mathrm{ml}$./minute. Electromyography at rest (see Fig. 3) and on effort has largely returned to normal. For the first time in four years the patient has obtained relief.

Case 2.-Mr. V.C., a butcher by trade, aged 53 years, was referred for investigation complaining of increasing stiffness of muscles over the previous six months. Up to this time he had been in perfect health. The stiffness began in the feet, spreading to the legs and later involved the arms, hands, and abdominal musculature. He noticed a hoarseness in his voice and stiffness of his tongue over this period. Associated with the stiffness there was a generalized weakness of all muscles. He had also noticed twitching in the muscles over the previous three months. He had had no difficulty with vision. Swallowing was occasionally difficult and at times the chest had become very stiff, leading to respiratory embarrassment on two occasions. The stiffness of the muscles first increased with effort, then improved with repetitive movements. He could then walk a short distance interrupted by occasional stiffening of muscles, paradoxically relieved by resting for a few seconds. The condition was growing worse. He lost $60 \mathrm{lb}$. in weight in six months.

There was no family history of muscle disorder and his five children were all well. The patient had recovered without complications from the common childhood illnesses and had remained well all his life. He had in the past consumed up to two bottles of brandy per week over a period of eight years, but had stopped drinking for the past three years. He complained of persistent sweating. The muscle stiffness was painless.

Examination revealed a middle-aged male with firm musculature which was in a state of continuous contraction. Fasciculation was noticeable in most muscles. The pulse was $100 /$ minute, blood pressure $110 / 76 \mathrm{~mm}$. Hg. The temperature fluctuated between 98.4 and $99.4^{\circ} \mathrm{F}$.

The respiratory, cardiovascular, and genito-urinary systems were normal. Palpation of the abdomen was difficult because of the muscle activity but there was no gross enlargement of the liver or spleen or abnormal masses. Rectal examination was negative.

The cranial nerves were intact, strenuous eye closure produced difficulty in opening the eyes, most noticeable after the second and third closure. All modalities of $\bar{Z}$ sensation were normal. Coordination was normal. The $\stackrel{\mathbb{D}}{\subseteq}$ muscles were in a state of constant contraction. Power was reduced in all muscles and in particular the extensor $\stackrel{\mathscr{C}}{\subseteq}$ muscles of the forearm. Tendon reflexes were impossible $\overline{\widehat{c}}$ to elicit, the plantar response was flexor. There was no $T$ myotonic response to percussion of the muscles including the tongue.

Special investigations as in Case 1 were essentially negative (see Table I), including barium studies of the gastrointestinal tract, an air encephalogram, and electroencephalography. The electromyographic studies were $\stackrel{\vec{\rho}}{\rightarrow}$ identical to those of Case 1. The patient was anaesthetized $\bar{C}$ with thiopentone $500 \mathrm{mg}$. in a $5 \%$ solution and maintained $\frac{\mathrm{O}}{\mathrm{O}}$ on oxygen and nitrous oxide. There was no change in $\overline{\bar{\omega}}$ muscle activity during this procedure. Succinylcholine in a dose of $500 \mathrm{mg}$. produced diminution and disappearance of all electrical activity within three minutes, lasting for 10 minutes. Curare in a total dose of $10 \mathrm{mg}$. produced $\overrightarrow{0}$ electrical silence after five minutes, lasting for a period of 40 minutes even though breathing began after a period of $\vec{\omega}$ 30 minutes. During this stage irritation of the muscle or movement of the limb failed to produce electrical activity. On a separate occasion a brachial block was performed using $25 \mathrm{ml}$. of $1 \%$ xylocaine; although the arm was is paralysed and anaesthetic, the motor activity persisted. This brachial block confirmed the findings of previous $\omega$ ulnar and median blocks which affected only the moter $\vec{\omega}$ supply to the small muscle of the hand.

It was decided to determine the sensitivity of the moto: end plate to curare. The purpose of this test was two-fola $\vec{Z}$ in that it might add further information to the fin elucidation of the abnormality and, secondly, might provide a therapeutic tool. The patient was prepared for a general anaesthetic and then given $1 \mathrm{mg}$. of curar. intravenously; three minutes later he complained generalized weakness which persisted for 10 minutes. $\vec{A} \mathscr{\sigma}$ further $1 \mathrm{mg}$. curare was then given and two minutes later $\rightarrow$ he began to have difficulty with breathing and swallowing, breathing becoming rapid and shallow. There was marked generalized weakness which completely overshadowed the reduction in the state of persistent muscle activity. Curare was therefore useless therapeutically, owing to $\frac{\mathrm{O}}{\mathrm{O}}$ the marked sensitivity at the motor end-plate to this @ blocking agent. Prostigmine was given to overcome the $\overrightarrow{\overrightarrow{0}}$ block but response was slow and even after $15 \mathrm{mg}$. had 3 been given intravenously slight generalized weakness $\supset$ persisted for the next hour. On another occasion, the patient was resistent to $1.5 \mathrm{mg}$. of decamethonium but developed generalized weakness after an additional 1.0 mg. had been given intravenously, which was not altered $\overline{0}$ by $20 \mathrm{mg}$. of edrophonium. The weakness was assessed $\overline{3}$ by the ulnar nerve stimulatory method described by Churchill-Davidson and Richardson (1952) and showed $\bigcirc$ a normal response with perhaps slightly resistant endplates.

Several muscle biopsy specimens were taken and revealed a similar histopathological picture to those of $\frac{D}{0}$ Case 1.

Arterial perfusions of the muscles of the forearm were $N$ carried out; there did not seem to be any increase in sensitivity to acetylcholine or potassium, but owing to the $N$ 
continuous spontaneous activity the results were difficult to interpret. There was, however, increased activity following on injection of both substances given in doses which would evoke contractions in normal subjects. Calcium and magnesium infusions produced some decrease in activity but this was associated with marked muscle weakness. The discharge was unaffected by atropine, quinine, cortisone, artane, adenosine triophosphate, thiamine diphosphate, or procaine amide.

Progress. - The condition steadily deteriorated over the next year, so that the patient became unable to walk and began developing contractures in the upper and lower limbs, producing flexion deformities at the elbows and wrists and per cavus respectively. There were several episodes of respiratory embarrassment and dysphagia.

After having exhausted the possibilities of deficient or excessive ions concerned in the maintenance of normal neuromuscular response, and having abandoned both blocking and depolarising agents as therapeutic tools owing to the occurrence of severe muscle weakness, sodium hydantoinate was used in an attempt to diminish the bizarre uncontrolled discharge from the lower motor neurone which characterizes this condition. The initial dose given was $100 \mathrm{mg}$. four hourly. A marked improvement occurred over the next two days and the drug was then reduced to a maintenance dose of $100 \mathrm{mg}$. six hourly. Six months later he was quite mobile and has been able to go back to his trade. The electromyography is vastly improved both at rest and on voluntary effort, the B.M.R. was +7 , and the oxygen consumption had fallen to $280 \mathrm{ml}$./minute after two weeks' therapy.

\section{Discussion}

There are a few pathological conditions which merit comment in that they have one or two features in common with this syndrome of continuous musclefibre activity. The similarities, however, are at the best only superficial.

Wechsler and Brock (1922) described a myostatic variant of dystonia musculorum deformans. In this disease, however, the myostatic manifestations were 'part of the disorder and always co-exists with the kinetic disturbance of which it is only a complement'. The postures assumed, muscle tone, and other manifestations of central nervous disorder bear no similarity to the cases with continuous muscle-fibre activity. Furthermore, as pointed out by Ramsay Hunt (quoted by Wechsler and Brock, 1922) this myostatic variety of torsion dystonia should be considered in the same light as paralysis agitans with tremor on the one hand and rigidity on the other.

McArdle (1951) described the case of a young adult male who complained of weakness, stiffness, and painful muscles after exercise. The muscles were of normal size and consistency at rest, but after exercise the respective muscles became hard and shortened, and developed areas of painful swelling. On resting the condition gradually im- proved and returned to normal. Passive extension of the affected muscles during this time met with considerable resistance and was painful. Electromyography revealed no activity at rest, normal action potentials during contraction and the painful areas of shortened muscle were electrically silent. McArdle compared the muscles in this case to the muscle in cases of iodioacetate and heavy metal poisoning and showed that the defect in his case was due to defective glycolysis. This was thought to be due to failure of the sulphydryl-splitting enzymes. McArdle gave his patient Dimercaprol with definite improvement, which was unfortunately shortlived. The defective glycolysis in McArdle's case has now been localized to a deficiency of phosphorylase, and the temporary improvement to Dimercaprol remains a mystery (McArdle, 1960). This and similar cases are obviously very different from the cases described in this paper which gave no evidence of defective glycolysis but are characterized by continuous muscle-fibre activity. The shortlived improvement following on Dimercaprol in Case 1 is, as with McArdle's case, difficult to explain.

Moersch and Woltman (1956) recorded 14 cases described as the 'stiff-man' syndrome. These cases were collected over a period of 30 years and included four women. The conditions appeared usually in the fifth decade with gradually increasing rigidity associated with intermittent painful spasms which could be precipitated by external stimuli or sudden voluntary or passive movement. The muscles first affected varied from those of the trunk, neck, and shoulders to those of the extremities, eventually spreading to involve all except the jaw muscles. The ultimate picture demonstrated marked involvement of the proximal musculature and to a lesser extent the distal. The muscles were described as rigid and board-like, fluctuating in severity. The tendon reflexes were brisk. An undetermined reducing substance was found in the urine of four cases, otherwise biochemical investigations were normal. Electromyographic studies in five cases gave a normal pattern. Muscle biopsy findings in two cases were normal. The conditions failed to respond to barbiturates, cortisone, antispasmodics, and relaxant drugs; 11 cases were followed for periods up to 15 years. There was a gradual deterioration in each. The severe proximal muscle involvement, the sparing of the jaw muscles, the brisk tendon jerks, the severe intermittent spasms with agonizing pain, the normal electromyographs and muscle biopsies, absence of fasciculation, increased B.M.R., and involuntary contraction of muscle fibres serve to distinguish the 'stiff-man' syndrome from the syndrome of continuous muscle-fibre activity.

There are some points of similarity between the 
cases with continuous muscle-fibre activity and the myotonic disorders of muscle. Continuous musclefibre activity is slightly aggravated by cold, a feature shared by a large number of myotonia sufferers. After strong voluntary contraction of any muscle there is a sustained shortening of the muscle lasting long after the initial stimulus has stopped, as in myotonia, though the mechanism is entirely different. In the former the shortening is maintained by persistent contraction, whereas in the latter it is a delay in relaxation. With continuous muscle-fibre activity the first movement is usually the most difficult to overcome but eases on repetition, whereas, at other times the first few movements are relatively free, becoming more difficult with repetition up to a point after which the movement becomes easier. The former progression is common in myotonia, and the latter, though rare, is occasionally seen and has been referred to as paradoxical myotonia. If the first movement, however, represents a maximum contraction, it is doubtful if this paradoxical progression exists. Marshall (1952) described a curious case of paradoxical myotonia in which the failure to respond to quinine or deteriorate with potassium, together with the absence of myotonic response to percussion makes one wonder whether this is in fact myotonia and not a variant of continuous muscle-fibre activity. The rates of discharge of electric activity vary between 5 and $250 / \mathrm{sec}$. which covers the myotonic range.

There are many points of distinction between these cases of continuous muscle-fibre activity and those with myotonia. Patients with myotonia are normal at rest, whereas those with continuous muscle-fibre activity show continuous muscular activity in both the waking and sleeping states. Electrical activity virtually ceases in myotonia with the onset of relaxation, with the exception of a low-voltage afterdischarge and the occasional phenomenon of reflex afterspasm (Denny-Brown and Nevin, 1941). The cases under discussion show continuous highvoltage electrical activity at rest, recruiting with voluntary effort, but on relaxation the activity continues for some time well above the abnormal pattern which was present before the voluntary contraction. The activity at rest is not affected by local blocking of the motor nerves. In contrast to myotonia, the activity of continuous muscle-fibre activity is abolished by blocking conduction at the motor endplates. The electrical activity at rest in continuous muscle-fibre activity does not have the appearance of normal motor unit potentials, but suggests bizarre spontaneous contraction of large numbers of muscle fibres. A notable feature in these cases is the lack of response to direct mechanical stimulation as opposed to that which occurs in the case of myotonia. These cases of continuous muscle-fibre activity have $\bar{q}$ obvious fasciculation involving all muscles in vary- $\stackrel{\mathbb{C}}{\bar{C}}$ ing degree, whereas fasciculation is rarely if ever seen in myotonia. In myotonia, the abnormal electrical response occurs only with the initiafo shortening of the muscle and not with sustainedes contraction, so that relaxation, though not neces- $\frac{\rho}{-}$ sarily complete, must be attempted before the myotonic discharges can be reproduced with further? movements of that muscle. Though aggravated $\vec{\Rightarrow}$ severely by contraction, patients with continuous $\frac{\text { f }}{?}$ muscle-fibre activity actively maintain a persistent 2 discharge at all times with the exception of a curious $\frac{\overline{\bar{n}}}{\mathrm{n}}$ period of dampening down of electrical activity approximately half a minute after a strong contrac- $\stackrel{\mathbb{2}}{0}$ tion and lasting for about 10 seconds. This dampening down is not a manifestation of fatigue and $\overrightarrow{0}$ might suggest that some product of muscle nerve metabolism or motor end-plate activity is responsible $\vec{\omega}$ for the apparent improvement.

Localization of the lesion is aided by noting that the persistent activity continues after interruption of the lower motor neurone by local nerve blockade. + . This would tend to take the lesion beyond the peripheral nerve, with the exception of the terming $\frac{\omega}{6}$ network which seems to possess, in addition to the응 conductive function of the nerve, certain peculiarition of its own and may be the site of abnormal electrical $Z$ discharges. The electromyographic studies woutd support the fact that the discharge is occurring frofn a site within the terminal branchings or the my neural junction itself.

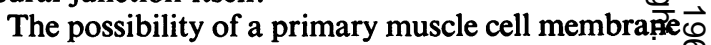
disorder is excluded by the response obtained with? myoneural blocking agents, barbituric acid derivatives, quinine, and the absence of irritability to needle electrodes or to percussion.

Evidence of abnormal function at the myoneural junction is shown by the increased sensitivity to the competitive blocking action of d-tubocurare. This finding suggests either excessive destruction of or an음 inadequate production of acetylcholine. Both these conditions are made improbable by the presence of continuous muscle activity, the fasciculation of the: muscles, the failure to respond to prostigmine or quinine, and the normal pseudocholinesterase level. An alternative explanation for this increased sen-윽 sitivity to curare suggests that the motor end-plate itself may have become relatively resistant as a resulto응 of the continuous acetylcholine stimulation, a phenomenon which was demonstrated by Brown? (1938) and more recently by Thesleff (1955). The $>$ response to calcium and magnesium may further indicate the presence of a somewhat resistant motor end-plate. When given parenterally these sub-stances produced marked weakness in dosages which $N$ 
did not affect normal controls. Magnesium suppresses the formation of acetylcholine in the nerve endings, while high calcium dosage increases the resistance of the motor end-plate (Brink, 1954). Carcinoma of the bronchus may be associated with extreme sensitivity to curare as shown by Eaton and Lambert (1957) so that unknown substances acting at the myoneural junction must also be kept in mind as a possible explanation for this finding.

Fasciculation may occur with lesions at the myoneural junction as is seen following the administration of depolarizing drugs. The electromyographic findings during this period resemble the discharge of continuous muscle-fibre activity, including the occasional motor unit potential formed by antidromic spread along the terminal branches as shown by Masland and Wigton (1940). With depolarization of the motor end-plate, however, there occurs a resistance to the action of blocking agents as opposed to the increased sensitivity in patients with continuous muscle-fibre activity.

Most of the evidence would favour an abnormal discharge from the terminal network of the motor neurone or myoneural junction with antidromic spread giving rise to fasciculation. The response to hydantionates in restoring these cases for practical purposes to normal would also favour an abnormal neuronal discharge rather than a metabolic defect at the myoneural junction.

Finally, in addition to these observations the modulating influence of the central nervous system must be considered. Lesions affecting central control may possibly result in the loss of specific inhibition affecting the production of mediating substances in the terminal network or myoneural junction, as was suggested for the lower motor neurone by Eccles (1957). Eccles was of the opinion that substances such as strychnine and tetanus toxin act by blocking an inhibitory transmitter substance in the lower motor neurone.

Considering the available information on these cases with continuous muscle-fibre activity, one concludes that a defect has been acquired at the level of the terminal network of the lower motor neurone, producing the bizarre and persistent neural activity which may be demonstrated electromyographically as a uncoordinated pattern of continuous muscle- fibre activity. This phenomenon is shown to be independent of transmission from the proximal part of the lower motor neurone and central nervous system though temporarily aggravated by voluntary movement.

The bizarre uncontrolled discharge from the terminal network has been restored to normal by the use of the anticonvulsant drug sodium hydantoinate which acts principally by increasing the sodium pumping action of nerve and muscle tissue as shown by Woodbury, Koch, and Vernadakis (1958).

\section{Summary}

Two cases demonstrating a syndrome of continuous muscle-fibre activity are presented and discussed, together with a therapeutic approach which has virtually restored normal mobility.

The syndrome is acquired, one case occurring in childhood and the other in middle age. The condition is separated from the group of myotonic disorders and some aspects of aetiology are discussed.

I wish to thank Dr. A. L. Agranat, senior physician, and Dr. F. K. Mills, medical superintendant, Johannesburg General Hospital, for their cooperation. Also Dr. D. Perk, Dr. Max Feldman, Dr. H. P. J. Pretorious, Dr. W. L. Vosloo, and Dr. K. I. Furman for referring the cases for investigation. Thanks are also due to $\mathrm{Mr}$. Keigh Allen and the Princess Nursing Home Neurosurgical Unit for the use of the electromyograph, Dr. D. Glauber for administering the anaesthetics, Dr. M. Berk for catheterizing the brachial arteries, and Dr. J. Kaufmann for the histopathological studies.

\section{REFERENCES}

Brink, F. (1954). Pharmacol. Rev., 6, 243.

Brown, G. L. (1938). J. Physiol. (Lond.), 92, 23 P.

Churchill-Davidson, H. C., and Richardson, A. T. (1952). Proc. roy. Soc. Med., 45, 179.

Denny-Brown, D., and Nevin, S. (1941). Brain, 64, 1.

Eaton, L. M., and Lambert, E. H. (1957). J. Amer. med. Ass., 163, 1117.

Eccles, J. C. (1957). The Physiology of Nerve Cells. John Hopkins Press, Baltimore.

Marshall, J.'(1952). J. Neurol. Neurosurg. Psychiat., 15, 206.

Masland, R. L., and Wigton, R. S. (1940). J. Neurophysiol., 3, 269. McArdle, B. (1951). Clin. Sci., 10, 13.

McArdle, B. (1960). Personal Communication.

Moersch, F. P., and Woltman, H. W. (1956). Proc. Mayo Clin., 31, 421.

Thesleff, S. (1955). Acta physiol. scand., 34, 218.

Wechsler, I. S., and Brock, S. (1922). Arch. Neurol. Psychiat. (Chicago), 8, 538.

Woodbury, D. M., Koch, A., and Vernadakis, A. (1958). Neurology, 8, Suppl. 1, p. 113 . 